\title{
The satellite positioning evolution in coastal processes
}

\author{
A. Angrisano, S. Gaglione, C. Gioia \& M. Vultaggio \\ Department of Applied Sciences, Parthenope University of Naples, Italy
}

\begin{abstract}
In order to guarantee future generations, a fair and consistent level of environmental quality is necessary to ensure sustainable development of coastal resources.

Adopting a static mode approach towards sea-level rise conflicts with sustaining a dynamic coastal system that responds to perturbations via sediment movement and long-term evolution. In the monitoring of this long-term evolution ancient technologies has played a very important role. Among these, satellite positioning is becoming more and more relevant.

This paper presents a brief survey of the evolution of GNSS (Global Navigation Satellite Systems) application in coastal process. The Global Position System (NAVSTAR GPS) is the best known of these satellite navigation systems. Global Navigation Satellite System (GNSS) is the standard generic term for satellite navigation systems that provide autonomous geo-spatial positioning with global coverage. A GNSS allows small electronic receivers to determine their location (longitude, latitude, and height) to within a few metres using the signals as transmitted by the GNSS satellites. In the same process the receivers also calculate the precise time of the signal reception and as such GNSS receivers can be used as highly accurate clocks.

Currently, the United States NAVSTAR Global Positioning System (GPS) is the only fully operational GNSS. The Russian GLONASS is a GNSS in the process of being restored to full operation. The European Union's Galileo positioning system is a next generation GNSS in the initial deployment phase. China has indicated it may expand its regional Beidou (also called COMPASS) navigation system into a global system. India's IRNSS, is also a next generation GNSS. However, its status and future is unclear as India seems to have entered into close cooperation with the Russians on the GLONASS system. Satellite Positioning, for this reason, is very popular and its accuracy can support a large
\end{abstract}


number of navigation and timing applications. However, it is generally recognized that these systems lack the accuracy, integrity, and availability to satisfy the more critical applications. This has led to the development of techniques to augment the basic GPS service.

The goal of this paper is to introduce an overview of the aforesaid technologies and to present the improvement outcome from their integration (multi constellation) and their augmentation (SBAS - Satellite Based Augmentation Systems) in terms of positioning accuracy; very important in the definition of long-term sediment movement and system integrity.

Keywords: GNSS, augmentation.

\section{Introduction}

The increasing attention paid to protecting the environment and the consequent need of mapping and surveying areas at risk, such as the coasts subject to erosion and sedimentation, have fostered the use of GNSS (Global Navigation Satellite Systems) systems in this area. Satellite navigation systems have not only revolutionized navigation, but also geodetic survey. By means of satellite range measurements, it is possible to obtain positioning accuracies previously impossible to obtain, especially for long baselines. With such systems it is possible both the control and the relief of the areas at risk, such as ridges subject to landslides. The advantage of GNSS systems is the simplicity of using them and speed of survey; in addition, along with the suitable augmentation techniques, they offer accuracy comparable to those reached by traditional means of survey.

\section{GNSS systems}

\subsection{GPS overview}

The Global Positioning System (GPS) is a space-based radionavigation system which is managed for the Government of the United States by the U.S. Air Force (USAF), the system operator; it is a dual use, military and civil system whose primary mission is to provide Position, Navigation and Time (PNT) services. GPS positioning is based on range measurement from known satellite positions to unknown user positions. The current GPS consists of three major segments: a space segment, a control segment and a user segment.

The space segment is a constellation of artificial satellites, 24 in the baseline configuration but more satellites can be in orbit [2], distributed in 6 orbital planes inclined at $55^{\circ}$ relative to the equatorial plane.

The control segment provides command, control, and maintenance services to the space segment and supports the PNT and the user segment is made up of all the system users, equipped with a GPS receiver-processor able to receive and decode the GPS signal to obtain PNT information.

GPS satellites broadcast signals on two carrier frequencies: L1 (1575.42 MHz) and L2 (1227.60 MHz). The carriers are modulated by two kinds 
of Pseudo-Random-Noise (PRN) codes, the Coarse-Acquisition (C/A) code with a $1.023 \mathrm{MHz}$ chip-rate and a period of one millisecond, and the Precise $(\mathrm{P})$ code with a $10.23 \mathrm{MHz}$ chip-rate a period of seven days. The C/A code is available on the L1 carrier only and the Precise (P) code on both L1 and L2.

PRN codes are referred to as "ranging code", because they are used to estimate the propagation interval of the signal from the satellite to the user (and hence the pseudo-range). The PRN codes are binary signals having spectral characteristic similar to random sequences, but actually are deterministic [4]. GPS system uses the Code Division Multiple Access (CDMA), i.e. all satellites use the same frequencies and each satellite transmits a different PRN code, so that at receiver level it is possible to distinguish the tracked satellite.

The C/A code is for civil use, it can be used by every user equipped with GPS receiver/processor and its use is associated with Standard Positioning Service (SPS). The P code instead is for military purpose, its access is restricted to authorized users only and its use is associated with Precise Positioning Service (PPS).

To meet the increasing demands of improved performance and to compete with the other developing GNSS systems, a GPS modernization program started in 2000 with the cancellation of the Selective Availability SA [11]. The program involves the deployment of new ground stations and the lunch of satellites of new generation able to broadcast additional signals. In detail, the new additional signals are a military signal (M-code) on both carriers, further codes on L1 carrier (L1C) and on L2 (L2C) for civil use and a third civil frequency L5.

The $\mathrm{L} 2 \mathrm{C}$ code is designed to meet commercial needs, allowing us to provide higher accuracy through dual-frequency ionospheric correction (associated to the existing C/A code on L1) [10]. L5 is designed to meet demanding requirements for transportation and safety-of-life. The satellites transmitting these types of signals are GPS IIF, whose $1^{\text {st }}$ launch was made 27 May 2010; 24 satellites of them are expected to be operational in 2018. L1C is designed with international partners for GNSS interoperability and will be transmitted by GPS Block IIIA satellites; 24 satellites of them are expected to be operational in 2021. Therefore, future civilian GPS users will be able to receive three signals on L1, L2 and L5.

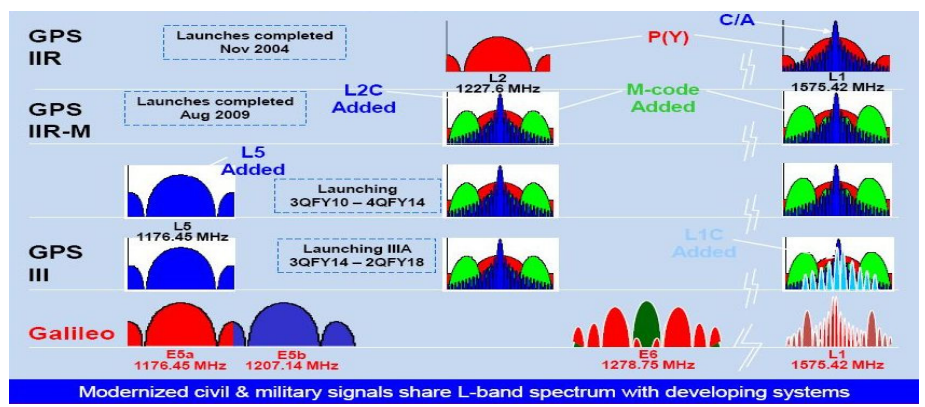

Figure 1: $\quad$ GPS signal modernization. 
L2C, together with the present L1 C/A-code signal and the future modernized civil signal L1C, will provide an alternative to augmented single frequency GPS for precision users. Many private and government precision applications could potentially benefit from multiple frequency GPS, e.g. centimeter accuracy is important to many land and marine surveying applications including planning, zoning, land management, cadastral surveying, harbor and port mapping, aids to navigation, coastal resources management, mapping and surveys of sensitive habitats.

\subsection{GLONASS overview}

GLONASS system has an operational principle similar to GPS system: the user equipment is able to measure pseudo-ranges and pseudo-range rates with respect to space vehicles, and uses this information to compute the position, velocity and for synchronization with a reference time scale.

Also GLONASS system consists of three segment as GPS, space, control and user segment, with similar tasks.

The space segment is a constellation of artificial satellites that, in the complete configuration are 24 , placed in three orbital planes whose ascending nodes are $120^{\circ}$ apart. Theoretically 8 satellites are equally displaced on each plane, with a displacement of argument of latitude among the planes of $15^{\circ}$. The orbits are planned to be circular with an inclination of $64.8^{\circ}$ and a radius of $19100 \mathrm{~km}$, corresponding to an orbital period of $11^{\mathrm{h}} 15^{\mathrm{m}}$ with ground tracks repeating every 17 orbital periods. The satellite constellation, if completely deployed, would provide a continuous and worldwide coverage for users on the Earth surface or in the near-Earth space [3]. The GLONASS space segment at November 11th 2010 includes 20 operating satellites, 4 in maintenance and 2 spares, and currently satellites of the "Glonass-M" generation are in orbit.

The Glonass-M satellites transmit signals on two frequency bands: L1 $1.6 \mathrm{GHz}$ and $\mathrm{L} 2 \sim 1.25 \mathrm{GHz}$. On both the bands, the carriers are modulated by two ranging codes, one associated to a standard positioning service accessible to all the users and another associated to precise positioning service restricted to authorized users only. The ranging code associated to the standard service has a $511 \mathrm{KHz}$ chip-rate and a period of one millisecond, the code associated to the precision service has $5.11 \mathrm{MHz}$ chip-rate.

GLONASS system uses the Frequency Division Multiple Access (FDMA) technique, i.e. each satellite transmits on carriers with different frequencies; hence at receiver level, GLONASS satellites can be distinguished by the frequency.

\subsection{Galileo overview}

The European Union (EU) conceived the establishment of Galileo in 1990s and it was finally approved in a meeting of the EU Transport Council in 2004. The first test-bed satellite system, GIOVE-A was launched in December 2005 and the second one, GIOVE-B was launched in April 2008. A series of in-space signal validations have been carried out and the ground control segment has been under 
development since that time. GIOVE-A and GIOVE-B are part of the first step of the In Orbit Validation (IOV) phase, including the use of a reduced constellation of four satellites to perform a validation of the system; four satellites are the minimum number necessary to perform positioning and timing.

The four Galileo In Orbit Validation (IOV) satellites will be launched in the following years and Galileo is expected to achieve its Full Operational Capability (FOC) in 2013. Once validation is complete, the full constellation will be deployed. The constellation will contain 30 satellites (27 operational satellites and 3 active spares) in three orbital planes, transmitting on three frequencies. The orbital planes are inclined 56 degrees relative to the equatorial plane. Due to the distribution of the satellites, Galileo will offer a slightly better coverage at high latitude which is very helpful for north European countries. In case of a single satellite failure, an active spare will be used to replace it, and therefore the user will not experience a significant degradation in overall performance [10].

Once complete, Galileo will offer four services:

- $\quad$ Open Service (OS), corresponding to the signals freely available which will be transmitted on two frequencies.

- Commercial Service (CS), corresponding to the encrypted signal which will be transmitted on three frequencies.

- Public Regulated Service (PRS) and Safety of Life (SoL), which will be used by the authorities for safety of life applications.

The Galileo Navigation Signals are transmitted in the four frequency bands. These four frequency bands are: the E5a band, the E5b band, the E6 band and the L1 band.

\section{GNSS augmentation}

Satellite Navigation systems are characterized by a set of parameters which define the system performance:

- Integrity, i.e. the ability to protect the user from inaccurate information in a timely manner.

- Accuracy, i.e. the difference between measured and true positions of a vehicle at any given time.

- Continuity, the ability to complete an operation without triggering an alarm.

- Availability, the ability to be used by the user whenever it is needed [13].

The GPS SPS does not provide suitable performance in terms of the defined parameters in all environment conditions or in every possible application. In severely signal degraded environments, e.g. mountainous or urban areas, GPS signals are blocked by buildings or natural obstacles and the positioning results inaccurate or even impossible owing to the lack of satellites [14]. Moreover GPS SPS is inadequate for critical safety applications like aircraft take-off or landing or for high-precision applications as land survey.

To solve GPS gap, different strategies can be adopted, e.g. the combined use of more satellite systems (as GPS/GLONASS or GPS/GLONASS/Galileo), the integration with other navigation systems (as GPS/inertial sensors) or the use of suitable techniques (DGPS, RTK). 


\subsection{DGPS}

In applications where greater accuracy is required, such hydrographic surveying, differential techniques (DGPS/Differential GPS) can be used to improve the GPS solution. In these areas, however, the surrounding topography or man-made structures may mask GPS signals line-of-sight. These obstructions may result in reduced satellite availability and reliability, degraded geometry, and so reduced horizontal DGPS accuracy. Relative positioning with GPS is achieved by performing simultaneous observation of satellite signal at two sites. The errors of the two receivers are spatially correlated, i.e. a particular satellite will experience common errors on ranging signal if the receivers are near.

The position of one receiver is precisely known and the common errors sources can be canceled or greatly reduced performing the difference between the observations; different approaches can be adopted and the more common are the double differences (DD) among the satellites and receivers, which removes all satellite dependent, receiver dependent and most of the atmospheric errors (if the distance between the two receivers is not too large).

A reference receiver, or base station used as a reference station (RS), computes corrections for the measurement of each satellite in view. Because individual pseudo-ranges must be corrected prior to the formation of a navigation solution, DGPS implementations require software in the reference receiver that can track all SVs in view and form individual pseudo-range corrections for each SV [5]. These corrections are passed to the remote, or rover, receiver which must be capable of applying these individual pseudo-range corrections to each SV used in the navigation solution.

Applying a simple position correction from the reference receiver to the remote one has limited effect on ranges used for positioning. Both receivers would have to use the same set of SVs measurement in their navigation solutions and to have identical GDOP terms (not possible at different locations) to be identically affected by bias errors [6].

The application of DGPS improves integrity compared to stand-alone GPS positioning. There are two types of DGPS approaches: Local Area DGPS (LADGPS) and Wide Area DGPS (WADGPS). LADGPS system uses only one ground reference station and transmits either scalar corrections or raw measurements to the near users. If the users are close to the reference station, common mode errors can be canceled and better accuracy can be achieved (of course the location of the reference receiver must be precisely surveyed) [7]. LADGPS broadcasts either corrections or raw measurements from the ground reference receiver to its users via any suitable data link. Because LADGPS is trying to cancel the common mode errors for the reference station and the user, accuracy strongly depends on their separation. As the user moves away from the LADGPS reference station, spatial decorrelation in the ephemeris errors, tropospheric and ionospheric errors cause accuracy degradation. The WADGPS approach uses multiple ground reference stations to monitor GPS satellites and generate vector corrections which include satellite clock errors, satellite ephemeris errors and ionospheric delays. Because of these vector corrections, 
WADGPS users will not suffer the spatial decorrelation problem characteristic of LADGPS. Approximately two meter accuracy (95\%) can be achieved over continental areas [8].

\subsection{RTK overview}

Centimetre-level accuracy positioning in real-time based on GNSS measurements was developed in the mid 1990s and is nowadays referred to as real-time kinematic (RTK) positioning. RTK is a special form of Differential GPS. The first use of GPS for centimeter position relied on static receivers which collected only carrier data, hours of data, to be post - processed. This was called static surveying.

Thanks to improvement in technology, the time computation for this kind of process decreases to a few minutes, having the so called, rapid-static technique. This one required only one static initialization of the receiver. From then, as long as the receiver maintained phase-lock, a centimeter accuracy is possible to achieve only in post processing. This was defined kinematic surveying. With faster and smaller computers, the desktop processing moved into the GPS receiver itself, providing real time results on field.

There are two kinds of RTK surveys: RTK with static initialization and RTK on-the-fly. Their difference leads only for the absence of the first part of the process procedure for the RTK on-the-fly technique.

However, for high precision applications, the use of carrier phase measurement must be used. This different type of data has an impact on the overall system complexity in terms of:

a) Equipment; the use of at least two double frequency receivers is necessary (for rover and base station). Also a data communication link is necessary (UHF or VHF) in order to broadcast this information between the receivers;

b) Computational complexity: this kind of measurement is, in fact, ambiguous, requiring sophisticated data processing software.

The limitation of single base RTK is the distance between reference and rover receivers. Such biases as: orbit error, ionospheric and tropospheric signal refraction depend from this distance. Good performance can be obtained if inter receiver length is less or equal to $10 \mathrm{~km}$. On the other hand, Wide Area Differential GPS (WADGPS) and the Wide Area Augmentation System (WAAS) use a network of master and monitor stations spread over a wide geographic area; measurement biases are modelled for any position of the area covered and proper transmitted. For this reason the positioning accuracy will be almost independent of the baseline length.

A good agreement between the above explained systems is the Network RTK; it is a centimeter-accuracy, real-time, carrier phase-based positioning technique capable of operating over inter-receiver distances up to many tens of kilometers with equivalent performance to current single base RTK systems (operating over much shorter baselines). The reference stations must be deployed in a dense enough pattern to model distance-dependent errors to such an accuracy that residual double-differenced carrier phase observable errors can be ignored in the context of rapid ambiguity resolution. Network RTK is therefore the logical 
outcome of the continuous search for a GPS positioning technique that challenges the current constraints of cm-accuracy, high productivity, carrier phase-based positioning [15].

\subsection{EGNOS overview}

Real-time satellite positioning at the sub-meter or even sub-centimeter level of accuracy requires the use of the augmentation system. DGPS has a fundamental limitation in that the broadcast corrections are good only for users in a limited area surrounding the base station. Outside this area the errors tend to be decorrelated, rendering the corrections less accurate. An obvious technical solution to this problem would be to use a network of base stations, each with its own communication link to serve its geographic area. However, this would require a huge number of base stations and their associated communication links.

Four space-based augmentation systems (SBASs) have been developed. These are the Wide-Area Augmentation System (WAAS), European Geostationary Navigation Overlay System (EGNOS), Multifunctional Transport Satellite (MTSAT) based Augmentation System (MSAS), and GPS \& GEO Augmented Navigation (GAGAN) by India. This SBAS are primarily defined as regional systems, and co-operation/co-ordination among the different systems will enable us to provide a seamless world-wide navigation system. To this aim, the systems have set up some common interoperability requirements and will provide adequate system compatibility. The development teams of SBAS systems meet regularly to develop and maintain common understanding of interoperability requirements and capabilities, and on the identification of the necessary interfaces among SBAS that each conceivable interoperability scenarios may imply [12].

EGNOS is the first step on the European contribution to the Global Navigation Satellite System. EGNOS is an augmentation system to the GPS and GLONASS Satellite Navigation systems, which provides and guarantees navigation signals for precision and kinematic applications.

EGNOS is composed of four segments:

- Ground segment

- Space segment

- User segment

- Support facilities.

The EGNOS Ground Segment consists of GNSS Ranging and Integrity monitoring Stations (RIMS) which are connected to a set of redundant control and processing facilities Mission Control Centre (MCC) that determines the integrity, differential corrections for each monitored satellite, ionospheric delays and generates GEO satellite ephemeris. This information is sent in a message to the Navigation Land Earth Station (NLES), to be uplinked along with the GEO Ranging Signal to GEO satellites; GEO satellites broadcast this data on the GPS L1 frequency with a modulation and coding scheme similar to the GPS one. All 
ground Segment components are interconnected by the EGNOS Wide Area Communications Network (EWAN).

The EGNOS Space Segment is composed of Geostationary transponders with global Earth coverage. The EGNOS AOC system is based on the use of the EGNOS GEO satellites AOR-E at $15.5^{\circ} \mathrm{W}$, ARTEMIS at $21.5^{\circ} \mathrm{E}$ and IOR-W at $25^{\circ} \mathrm{E}$, and the ESA ARTEMIS navigation transponders.

The EGNOS User Segment consists of an EGNOS Standard receiver, to verify the Signal-In-Space (SIS) performance, and a set of prototype User equipment used to validate and eventually certify EGNOS for the different applications being considered.

EGNOS support facilities include the Development Verification Platform (DVP), the Application Specific Qualification Facility (ASQF) and the Performance Assessment and System Checkout Facility (PACF). Those are facilities needed to support System Operations and future Qualifications.

\section{Conclusions and results}

The goal of this work is to verify and to compare the satellite positioning Augmentation Systems discussed. This study is focused on a measures accuracy analysis where the reference solution for comparison, computed by a post processing geodetic method (DGPS), is:

Table 1: $\quad$ WGS84 reference coordinate.

\begin{tabular}{|c|c|c|}
\hline Latitude & Longitude & Height \\
\hline $40^{\circ} 49^{\prime} 24.37413^{\prime \prime} \mathrm{N}$ & $14^{\circ} 12^{\prime} 58.11149^{\prime}{ }^{\prime} \mathrm{E}$ & $122.659 \mathrm{mt}$. \\
\hline
\end{tabular}

24 hours of data were collected, at $1 \mathrm{~Hz}$ frequency, in the Parthenope University ECDN (ESV/The Data Collection Network) Station on November $30^{\text {th }} 2010$ by a Septentrio PolaRx 2 receiver. These data are processed by PEGASUS v 4.5.0 software, developed by EUROCONTROL, currently considered as a benchmark from the European research community involved in the same network.

Accuracy and precision indicate different attributes on how close a solution is to the reference. Accuracy refers to the ability of a system to be close to the reference value, while precision refers to the ability of the system to repeat its solution. In the Gaussian distributions domain, good accuracy would correspond to a zero mean, while high precision would correspond to a low standard deviation. Thus it is possible to have a precise, but inaccurate system (and vice versa), when the distribution is centered on a bias with a very small deviation [16].

In the following figures the solution obtained by a GPS stand alone processing method is shown. 


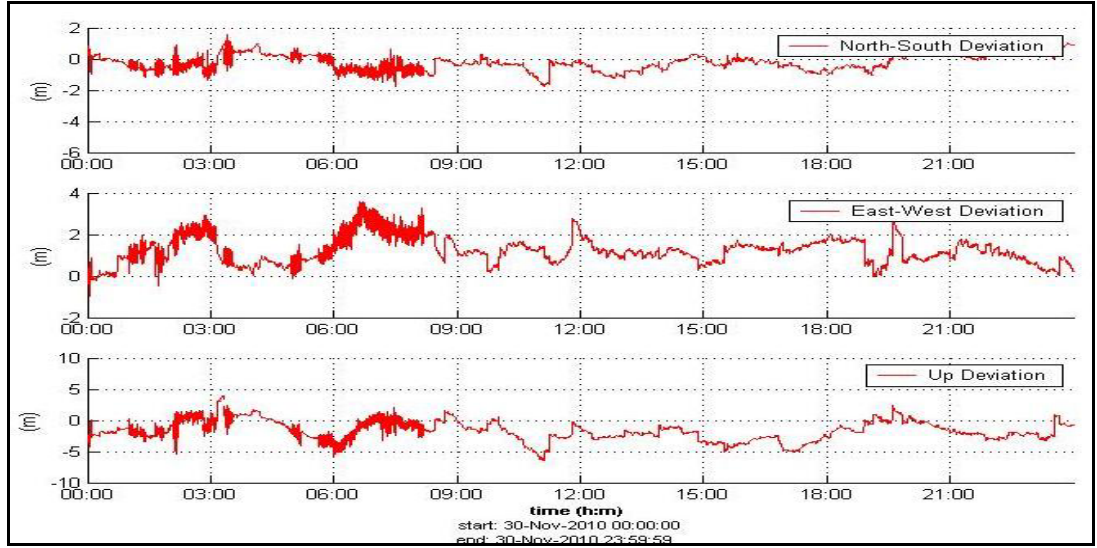

Figure 2: Deviation from the reference coordinate with GPS stand-alone.

The distribution of the error is:

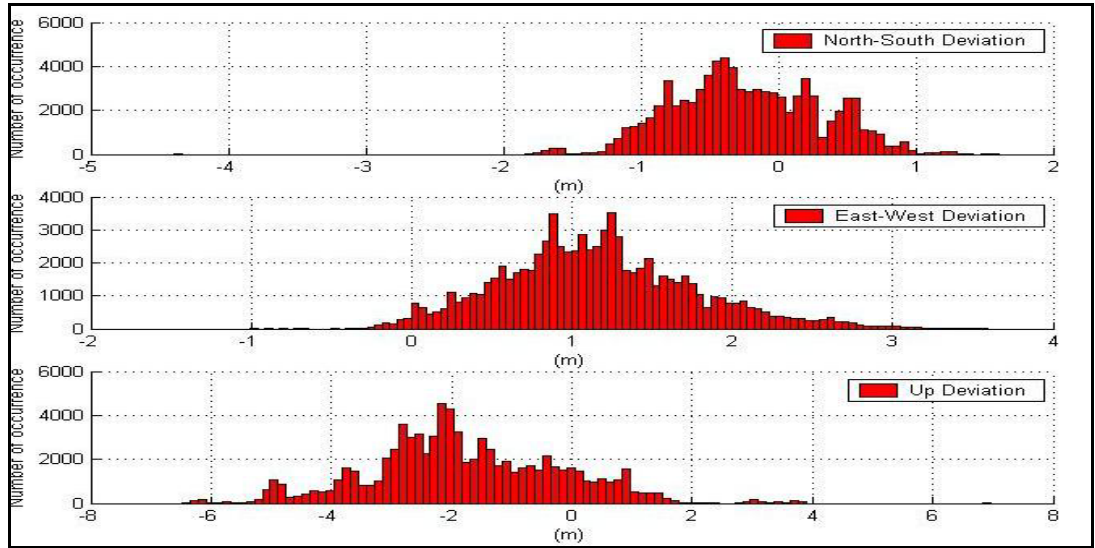

Figure 3: $\quad$ Noth Est Up deviation with GPS stand-alone.

In the following figures the solution obtained by GPS measures augmented by EGNOS correction is shown.

Figure 5 shows how the augmented solution is more accurate than the GPS stand alone one. From a qualitative analysis it is clear that in this case the error distribution is closer to the zero mean and from a quantitative analysis (see table 2) the precision is also improved. 


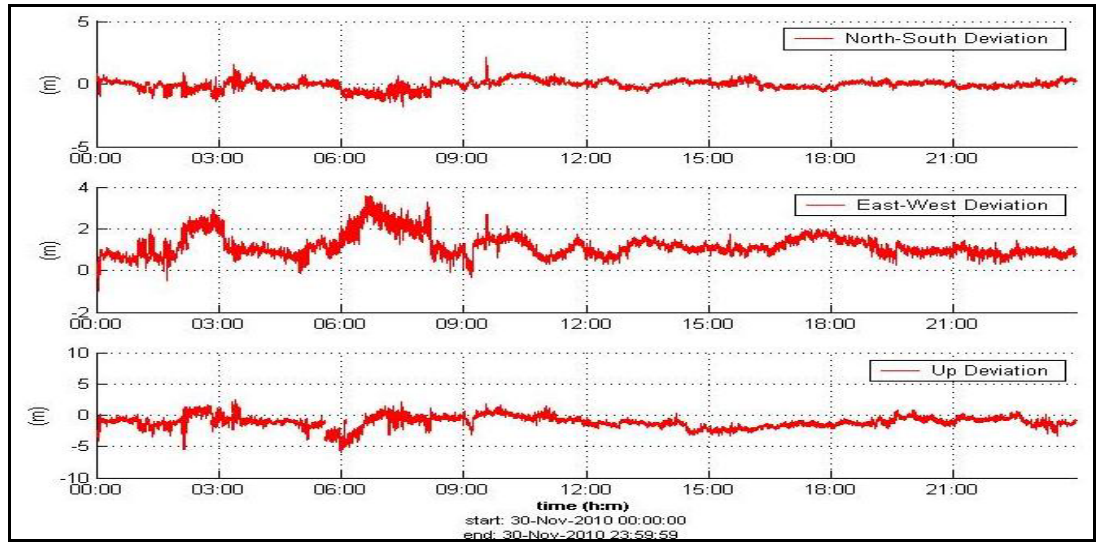

Figure 4: Deviation from the reference coordinate with SBAS.

The distribution of the error is:

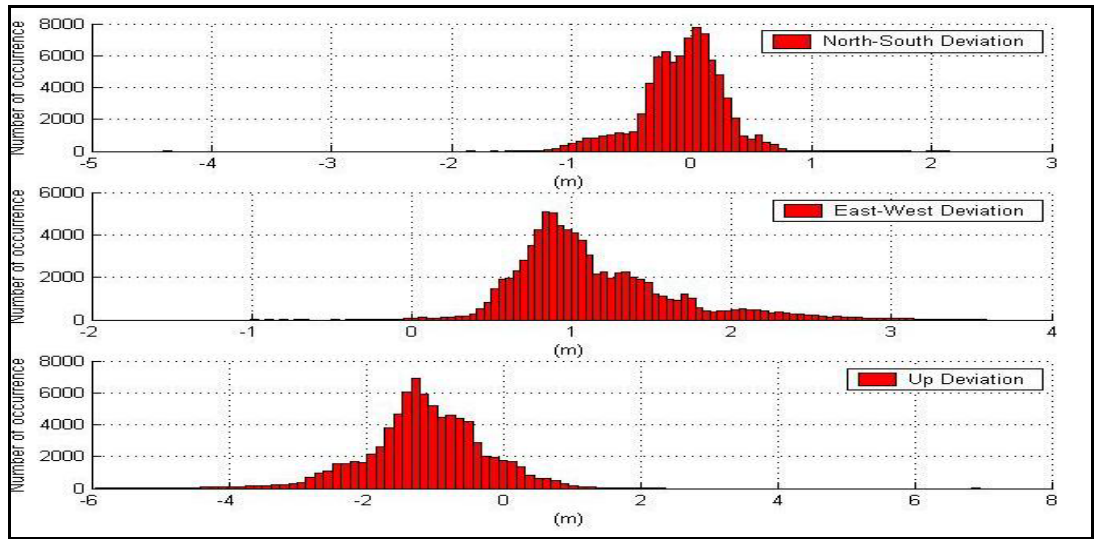

Figure 5: $\quad$ Noth Est Up deviation with SBAS.

The accuracy and precision of each result are summarized in Table 2.

Table 2: $\quad$ Error distributions standard deviation and variance.

\begin{tabular}{|l|c|c|c|c|c|c|}
\hline Method & $\begin{array}{c}\text { Std_N/S } \\
{[\boldsymbol{m}]}\end{array}$ & $\begin{array}{c}\text { Std_E/W } \\
{[\boldsymbol{m}]}\end{array}$ & $\begin{array}{c}\text { Std_U/D } \\
{[\boldsymbol{m}]}\end{array}$ & $\begin{array}{c}\text { Var_N/S } \\
{[\mathbf{m}]}\end{array}$ & $\begin{array}{c}\text { Var_E/W } \\
{[\boldsymbol{m}]}\end{array}$ & $\begin{array}{c}\text { Var_U/ } \\
\boldsymbol{D}[\boldsymbol{m}]\end{array}$ \\
\hline GPS & 1.0158 & 1.2894 & 2.2134 & 1.0319 & 1.6625 & 4.8994 \\
\hline SBAS & 0.7226 & 0.7031 & 1.0949 & 0.5222 & 0.4944 & 1.1988 \\
\hline
\end{tabular}


The most important improvement obtained by the application of the SBAS correction leads in the Height solution that is of great interest within the geodynamic study.

The good GPS stand-alone solution is due to the optimal measurement condition. Infect, as aforesaid, it was processed data from a static receiver in a good location (open sky). A next step of this research it will be the improvement assessment in worst condition (urban canyon, non static mode).

\section{References}

[1] IS-GPS-200 (2004) Navstar GPS Space Segment/Navigation User Interfaces, Revision D, ARINC Research Corporation, El Segundo, CA.

[2] Global Positioning System Standard Positioning Service Performance Standard (2008), US Department of Defense, 4th Edition, September 2008.

[3] ICD-GLONASS (2008) Global Navigation Satellite System GLONASS Interface Control Document, version 5.1, Moscow.

[4] Kaplan, E.D. (2006), Understanding GPS: Principles and Applications (second edition), Artech House Mobile Communications Series.

[5] Tunissen, P.J.G. Kleusberg A. (1998), GPS for Geodesy. Springer, Germany.

[6] Skaloud J. (1999) Optimizing Georeferencing of Airborne Survey System by INS/DGPS, PhD Thesis, University of Calgary, Alberta.

[7] Zhang Y. (2005) High performance differential global positioning system for long baseline application, $\mathrm{PhD}$ Thesis, Ohio University.

[8] Differential Global Positioning System (DGPS) for Flight Testing.

[9] Galileo Open Service Signal In Space Interface Control Document (OS SIS ICD). (2006)

[10] Cao W. (2009) Multi-frequency GPS and Galileo Kinematic Positioning with Partial Ambiguity Fixing, University of Calgary, Alberta.

[11] Zhang W. (2009) Triple Frequency Cascading Ambiguity Resolution for Modernized GPS and GALILEO, University of Calgary, Alberta.

[12] Mohinder S. Grewal, Global Positioning System, Inertial Navigation, and Integration. Second edition Wiley (2007).

[13] M.G. Petovello, G. Lachapelle, P.C. Fenton, (31st Annual Precise Time and Time Interval Meeting), an Overview of GPS Augmentation Systems.

[14] Angrisano, A., Gaglione, S., Pacifico, A. and Vultaggio M. (2009): MultiConstellation System as Augmentation to GPS Performance in Difficult Environment or Critical Applications, Proceedings ENC-GNSS 09, Naples, Italy, 3-6 May 2009.

[15] Van Diggelen F., GPS and GPS+GLONASS RTK, ION-GPS, September 1997.

[16] Bancroft J. (2007) A Study of Accuracy Metrics (ENGO 585). 